\title{
Suggestion pour une prise d'eau automatique de montagne. Détecteur à gravier ${ }^{(1)}$
}

\section{Suggestion for an automatic intake in mountainous regions Gravel detector}

\author{
PAR. J. MOLBERT, L. VULLIERME ET J. ZUMSTEIN
}

ÉLECTRIGITÉ DE FRANCE. R.E.H. ALPES-II

\begin{abstract}
Les tendances actuelles de la technique des chutes d'eau en haute montagne nous conduisent à grouper un grand nombre de petites prises d'eau pour alimenter un grand réservoir qui dessert une usine unique importante. Dans ces conditions le prix de chacune des prises intervient peu dans l'économie de l'ensemble du projet. Ce sont alors les conditions d'exploitation - entretien du matériel, personnel de surveillance, pertes d'eau, etc. - qui régissent la conception des prises. C'est dans cet esprit qu'on a été amené à mettre au point un type de dégraveur discontinu automatique, muni d'un détecteur d'engravement et éventuellement d'un « mur plongeant » qui équipera la plupart des prises d'eau de l'aménagement de Roselend.
\end{abstract}

\begin{abstract}
The present day trend in harnessing water power in mountainous regions is to group together a large number of small intakes to feed a large reservoir which serves one large power station. Under these conditions the cost of each intake plays but a small part in the economy of a scheme as a whole. It is therefore factors such as maintenance of equipment, inspection staff and water losses which govern intale design. Such considerations led to the design of an automatic, discontinuously operating gravel remover which incorporates a gravel filling detector and, if necessary, a "plunging wall". This device will be used on most of the intakes for the Roselend project.
\end{abstract}

\section{1. - INTRODUCTION}

\section{1. - Considérations sur les prises d'eau de haute altitude des aménagements hydroélectriques.}

Dans les aménagements hydro-électriques du type alpin, l'utilisation plus poussée des sites, en multipliant les prises d'eau secondaires, confère à ces derniers ouvrages, quoique d'importance

(1) Couvert par un brevet. parfois modeste, un rôle de premier plan dans la bonne marche d'une installation.

Souvent, ces prises servent à l'alimentation d'un réservoir; la valeur plus élevée de l'eau captée fait alors apparaître comme d'autant plus rentable la recherche d'un fonctionnement sûr et d'un rendement élevé. De simples améliorations de détail apportées à ces ouvrages et une meilleure définition des consignes d'exploitation permettent, dans beaucoup de cas, de résorber un déficit de production de plusieurs millions de $\mathrm{kWh}$. 


\section{2. - Problème du dessablage ou dégravage.}

Le principal problème à résoudre est évidemment la question du dégravage, car les torrents de haute altitude charrient en général énormément de cailloux, sables et boues; le dégravage (ou dessablage) peut se faire en gros de deux manières :

- soit par purge continue,

- soit par purge discontinue.

Les diverses études et observations que l'on a pu faire nous ont montré qu'il était préférable, pour ce genre de prise, d'adopter des chasses discontinues qui simplifient beaucoup les ouvrages de chasse proprement dits et entraînent, d'une facon générale, des pertes d'eau beaucoup moins importantes.

\section{3. - Description d'une prise d'eau de haute montagne.}

Avant de commencer la description du détecteur à gravier, il convient de dire quelques mots de l'ensemble prise d'eau-dégraveur sur lesquels il pourra être adapté (fig. 1).

- Prise d'eau proprement dite:

Elle est, en général, du type en dessous, c'està-dire formée d'une simple fosse creusée au mi- lieu dụ torrent et recouverte, à sa partie supérieure, de grilles grossières.

\section{- Dégraveur :}

L'eau est ensuite dirigée sur un canal assez profond qui sert de fosse de décantation. Ce canal est obturé par une vanne à secteur qui sert à faire des chasses périodiques pour évacuer les graviers et sables qui se sont déposés. Une fois décantée, l'eau se déverse, par l'intermédiaire d'un déversoir latéral D, dans la galerie d'amenée.

\section{- Vanne de chasse :}

La vanne de chasse qui obture le canal de décantation est du type à secteur hyper-équilibré; elle est prolongée par un contrepoids d'un poids tel que la vanne seule aurait tendance à s'ouvrir (fig. 1). Ce contrepoids est équilibré par un flotteur situé dans un puits et il est calculé de telle manière que, si le puits est vide d'eau, il maintient par son poids la vanne fermée. Au contraire, si le puits est plein d'eau, la poussée d'Archimède suffit pour le soulever et la vanne s'ouvre. Ce système permet donc de lever la vanne sans effort manuel en remplissant d'eau le puits du flotteur. Le principe de ces vannes est d'ailleurs bien connu et a fait ses preuves depuis longtemps.

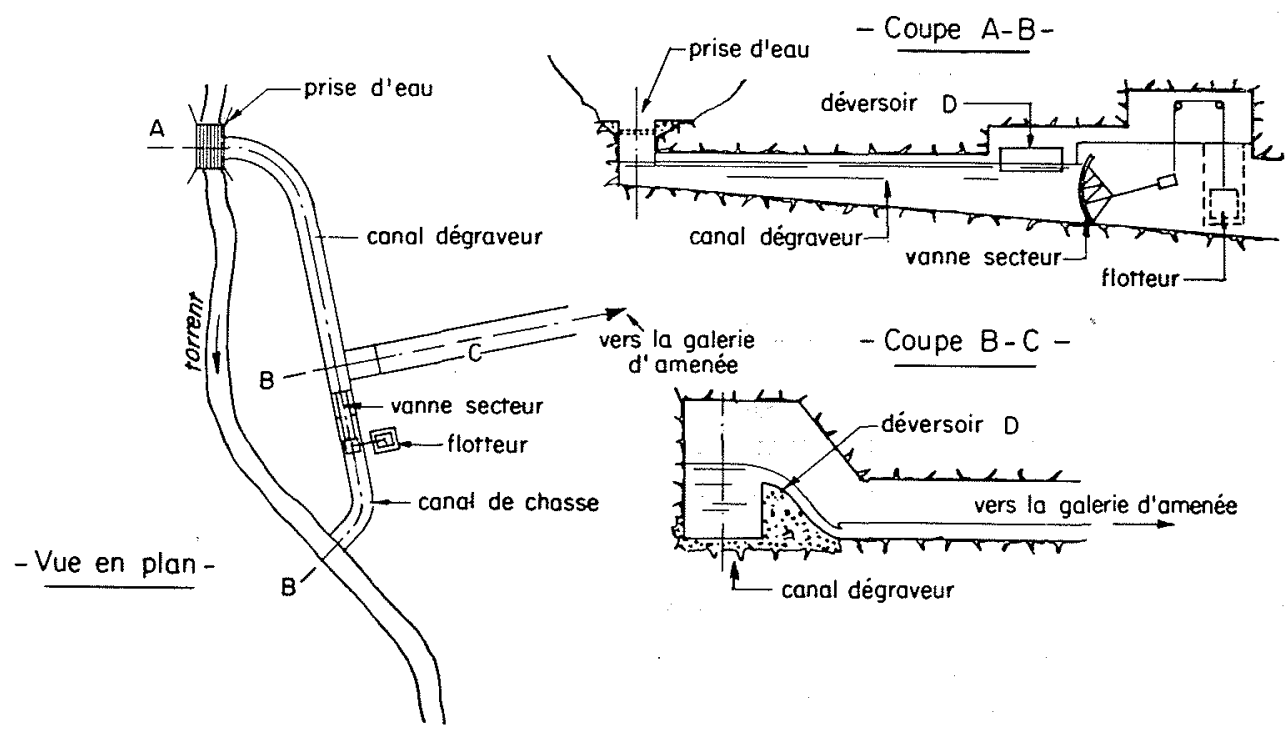

FIG. 1 


\section{2. - PROBLEME DU GARDIENNAGE ET DES PERTES D'EAU}

\section{1. - Problème du gardiennage.}

Un aménagement complexe, type Aussois ou Roselend, comprend un grand nombre de petites prises d'eau situées souvent dans des endroits d'accès très difficile (sinon impossible à certaines périodes de l'année). D'autre part, étant donné l'importance des transports solides pendant les périodes de hautes eaux, ces prises d'eau nécessitent une surveillance quasi-permanente.

On conçoit donc, dans ces conditions, la difficulté que représente le problème du gardiennage. En période de crues, on sera presque obligé d'avoir un gardien par prise; en étiage, au contraire, l'eau étant propre, le gardiennage est superflu. Il semble donc particulièrement rentable d'imaginer des systèmes permettant de se passer de gardien ou, tout au-moins, de limiter d'une manière sensible le nombre de visites aux prises, de telle manière qu'un gardien puisse exploiter à lui seul un grand nombre de prises d'eau.

Dans ce but, la région Alpes-II a mis au point un système dit " horloge hydraulique » qui a été décrit en son temps dans la Houille Blanche, $n^{\circ} 6,1955$, pp. 875-878, et dont nous allons donner une description sommaire.

\section{2. - Description sommaire de l'horloge hydraulique.}

\subsection{1. - Description :}

Ce système comprend deux fosses de volume utile égal A et B reliées par un siphon; la fosse B n'est pas autre chose que la fosse du flotteur de la vanne de chasse dont nous avons parlé plus haut. La fosse A se remplit lentement; quand elle est pleine, le siphon s'amorce et la vide entièrement dans B, provoquant la montée complète de la vanne à secteur et une chasse; la vanne d'évacuation $\mathrm{C}$ permet de régler la durée de cette chasse (fig. 2).

\section{Remplissage de la fosse $A$}

Le niveau dans le canal de décantation est réglé par le déversoir D. La fosse A est en communication avec le canal par un jeu d'orifices calibrés $\mathrm{E}$. Le volume de $\mathrm{A}$ et la disposition des orifices $\mathbf{E}$ permettent de choisir une loi réglant le nombre de chasses en fonction du débit.

\subsection{2. - Références. - Le Pingon :}

Ce système a fonctionné pendant trois saisons au Pingon où il a donné à l'exploitation toute satisfaction. Le Pingon est un torrent d'origine glaciaire qui, par conséquent, transite une assez grande quantité de matériaux solides.

Pendant trois ans, cette prise d'eau a été simplement visitée par le gardien qui n'a eu à s'assurer que de sa bonne marche; aucune panne sérieuse n'a été constatée. Une prise d'eau de ce type, si elle est bien réglée, permet done de se passer presque complètement de gardien.

\section{3. - Problème des pertes d'eau. - Détec- teur à gravier.}

L'horloge hydraulique répond donc bien au but recherché : se passer complètement de gardien. Cependant, la principale difficulté du système est de se donner une loi : fréquence des chasses-débit du torrent. Pratiquement, on règle l'appareil de manière à faire un nombre de chasses bien plus grand qu'il ne serait strictement nécessaire, ce qui conduit à des pertes d'eau. Par sécurité, il convient de faire grosso modo deux fois plus de chasses qu'il n'est nécessaire.

L'idéal serait évidemment de ne faire des chasses que lorsque le dégraveur est plein. Le détecteur à gravier a été imaginé dans ce but. C'est un appareil qui se met à la place de l'hor-

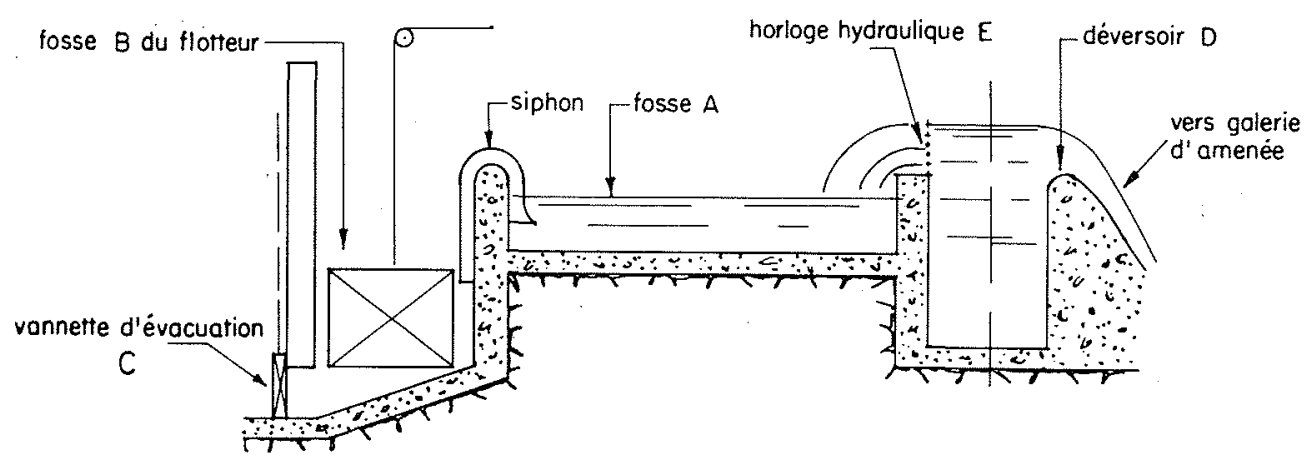

Fic. 2 
loge hydraulique proprement dite et qui permet de ne remplir la fosse A du système précédemment décrit que lorsque le dégraveur est plein de gravier et de sable.

En conclusion, un dégravage continu dépensera environ $10 \%$ dụ débit dérivé des hautes eaux, soit pour Roselend, 20 millions de mètres cubes ou 50 millions de $\mathrm{kWh}$, ce qui représente une perte actualisée de plus de 2 milliards de francs. L'adoption de l'horloge hydraulique permettrait de réduire cette perte à moins de $1 \mathrm{mil}$ liard, et le détecteur à gravier moins de $1 / 2$ milliard, ces chiffres n'ayant, bien entendu, qu'une valeur d'ordre de grandeur très approximative.

\section{3. - DETTECTEUR A GRAVIER}

\section{1. - Principe (fig. 3).}

Cet appareil est en somme une amélioration de l'horloge hydraulique. On retrouve les deux fosses $A$ et $B$ précédemment décrites, mais la fosse A, au lieu de se remplir lentement, se remplira rapidement au moment où le dégraveur. sera plein pour faire une chasse. ties :

Il se compose schématiquement de deux par-

- Le détecteur proprement dit, qui est placé dans la galerie de décantation. d'eau dans le vase est pratiquement le même que le niveau dans le dégraveur.

Ce flotteur est relié à une vannette pouvant mettre en communication le canal de décantation et la fosse $A$. Il est réglé de telle manière que lorsque le vase est plein d'eau, la vannette est fermée; lorsque le vase est vide, la vannette est ouverte et l'eau du canal de décantation se déverse dans la fosse $A$.

Le détecteur marche donc de la manière suivante : quand le canal de décantation se remplit de sable, l'extrémité du tube se bouche et le

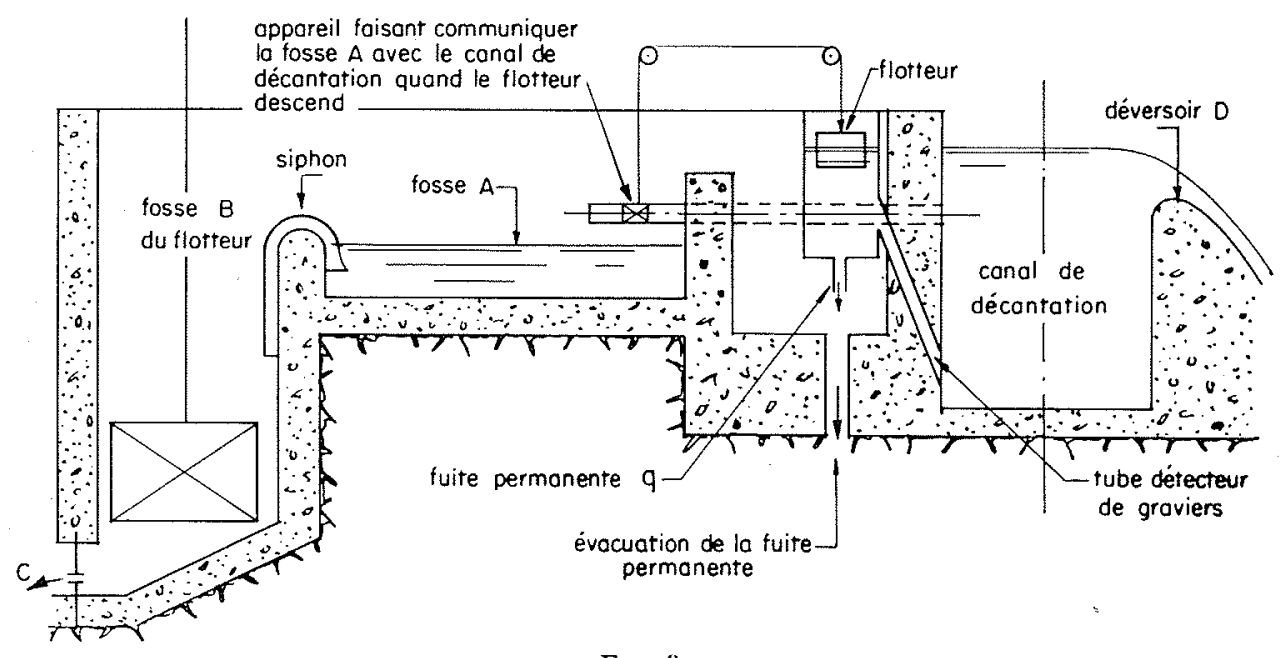

FIG. 3

- L'appareil servant à remplir la fosse A quand le sable a été détecté, lui-même composé de deux appareils :

- le vase et le flotteur,

- la vannette de communication.

Un tube (le détecteur proprement dit) met en communication le radier du dégraveur avec un vase dans lequel se trouve un flotteur. Ce vase est percé d'un trou provoquant une petite fuite permanente $q$. Cette fuite étant faible, le niveau niveau dans le vase s'abaisse à cause de la fuite permanente $q$ qui n'est plus alimentée. Le flotteur suit le mouvement et provoque l'ouverture de la vannette mettant en communication le dégraveur avec la fosse $A$; cette dernière se remplit. Quand elle est pleine, le siphon s'amorce et provoque une chasse comme pour l'horloge hydraulique précédemment décrite. La chasse dans le canal de décantation provoque le nettoyage et le débouchage de l'orifice du détecteur. Une fois la 


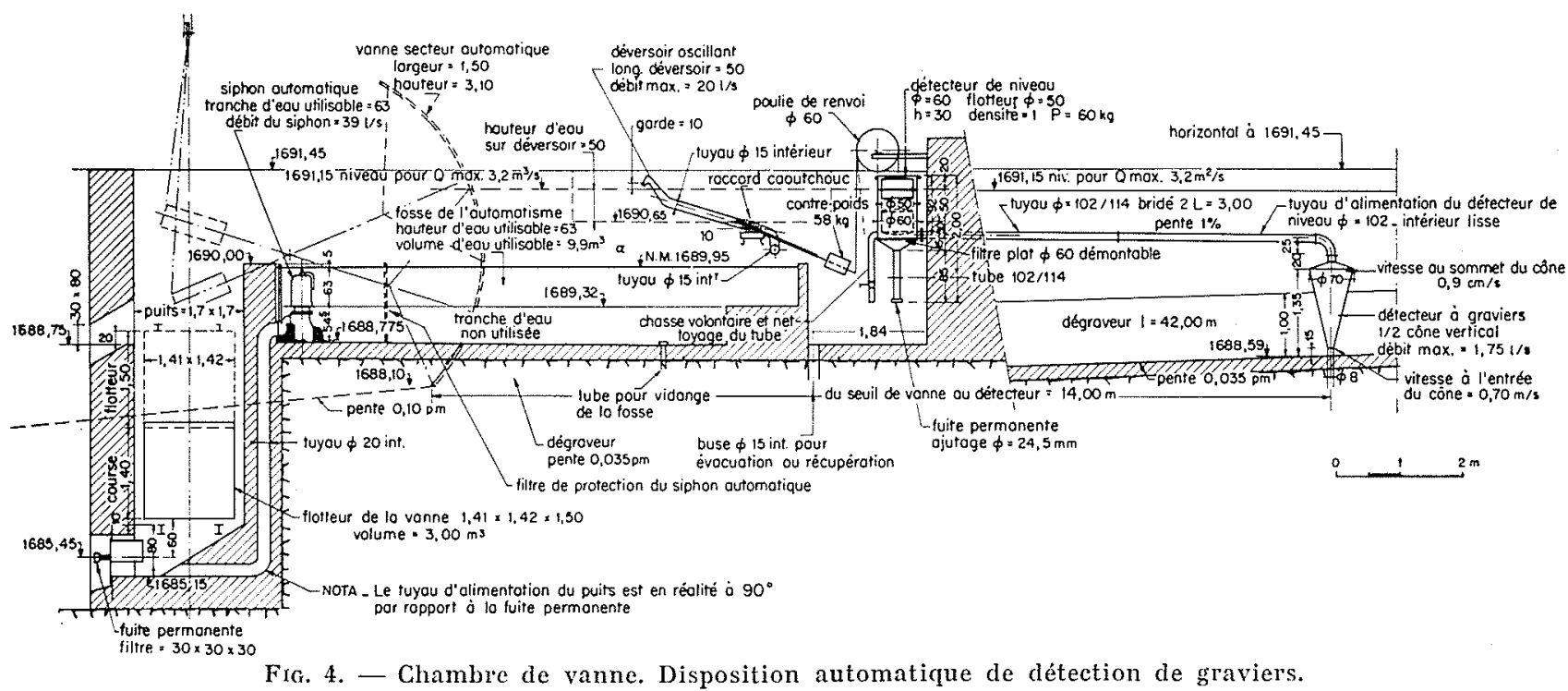

chasse terminée, le canal se remplit d'eau à nouveau, le vase dụ flotteur également, ce qui provoque la montée du flotteur et la fermeture de la vannette de.communication. L'appareil est donc revenu à son état initial, prêt à fonctionner à nouveau dès qu'il y aura des dépôts solides dans le canal de décantation.

Ceci est le principe du système; en réalité, sa mise au point définitive nous a conduits à luj faire subir de nombreuses modifications. En particulier :

- le détecteur n'est plus un simple tube, mais une sorte de petit dessableur indépendant,

- la vannette de communication a été remplacée par un appareil que nous avons appelé " déversoir oscillant » et qui a l'avantage d'avoir une très grande sensibilité et de fonctionner sur une différence de niveau entre vase et canal plutôt que sur une baisse de niveau en valeur absolue.

\section{2. - Description détaillée.}

3.2.1. - Détecteur proprement dit (fig. 5) :

Il est constitué par une sorte d'entonnoir en lorme de demi-cône posé verticalement sur sa
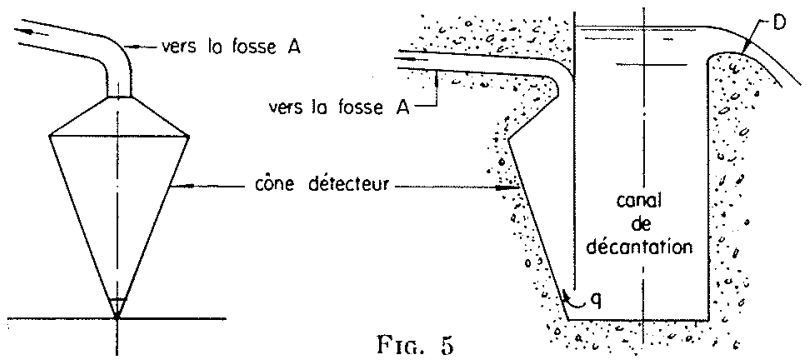

pointe contre une des parois du canal de décantation (fig. 5).

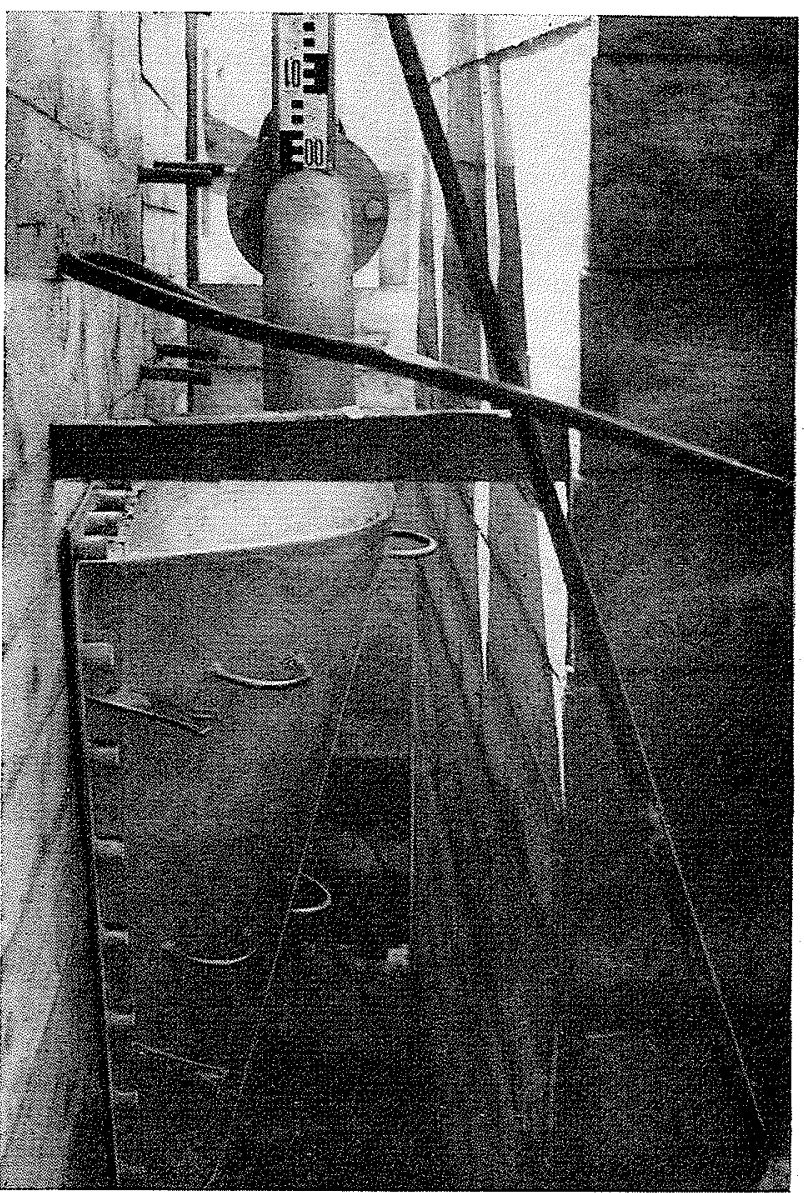

Pнoтo 1. - Vue du cône détecteur. 


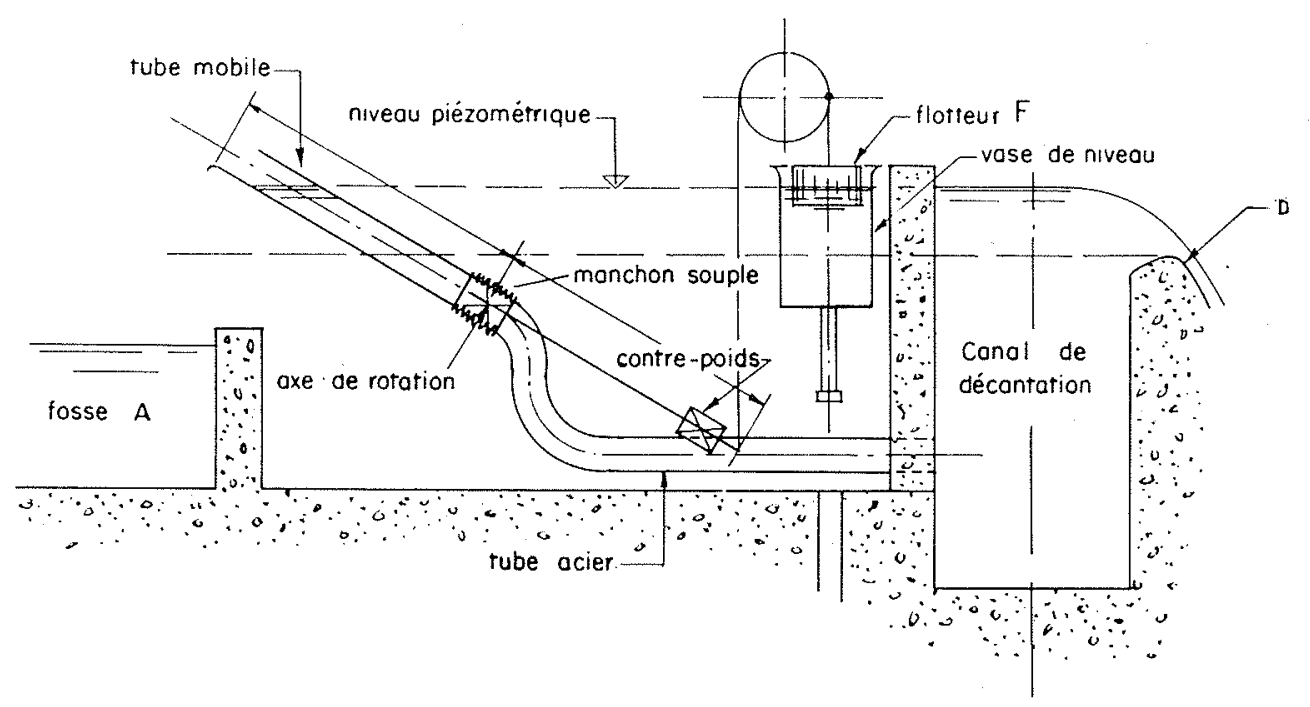

FIG. 6

Cet appareil est en somme une sorte de petit dessableur. Dans le bas, l'eau entrant a encore une vitesse suffisante pour entrainer les sables et graviers; cette vitesse, diminuant progressivement vers le haut du cône, n'est plus suffisante pour entraîner les matériaux solides qui retombent et finissent obligatoirement par boucher le bas du cône, en formant en quelque sorte bouchon.

Ce cône détecteur est relié au vase du flotteur par un tuyau métallique de pente montante vers le vase et de longueur variable suivant la position relative des deux appareils.

\subsection{2. - APPareils SERVANT a REMPlir la FOSSE A QUAND LE SABLE A ÉTÉ DÉTECTÉ (fig. 6).}

\subsubsection{1. - Vase et flotteur :}

Le vase est un simple tube cylindrique de $600 \mathrm{~mm}$ de diamètre, bien visible sur le plan. Il est prolongé à sa partie inférieure par un tube de $100 \mathrm{~mm}$ de diamètre à l'extrémité duquel se trouve un orifice exactement calibré pour la fuite permanente $q$.

Le flotteur a pour densité 1 et un volume de 50 litres. Il est relié au contrepoids du déversoir oscillant par un câble souple en acier inoxydable passant sur une poulie à gorge de $600 \mathrm{~mm}$ de diamètre.

\subsubsection{2. — Déversoir oscillant (fig. 6) :}

Pour simplifier notre exposé, nous avons écrit plus haut que le flotteur situé dans le vase était lié à une vannette pouvant mettre en communication le canal de décantation avec la fosse $A$.
En réalité, un tel système marche difficilement à cause des différences de niveau dans le canal, dues aux variations de débit dérivé; en effet, le niveau dans le canal est donné par la lame déversante sur le déversoir $\mathrm{D}$; plus le débit dérivé est important, plus l'épaisseur de la lame est grande. Pour que l'appareil marche en toutes circonstances, c'est-à-dire quel que soit le débit dérivé dans la prise, il faut donc que la vannette ne s'ouvre que lorsque le niveau dans le vase a baissé au-dessous du niveau du déversoir D. Donc, à faible niveau dans le dégraveur, l'appareil est très sensible et se déclenche rapidement; au contraire, à niveau haut, l'appareil manque totalement de sensibilité.

Il faut donc trouver un système ayant une sensibilité indépendante du débit dérivé dans le canal, autrement dit un appareil détectant une différence de niveau entre vase et canal, et non un niveau comme dans le cas de la vanne et du flotteur.

C'est ce système que nous avons appelé déversoir oscillant et qui se trouve schématisé sur la figure 6 .

Ce déversoir oscillant est un balancier posé sur couteau, dont un des bras est constitué par un tube de $150 \mathrm{~mm}$ de diamètre terminé en forme de déversoir, et l'autre bras par une simple tige rigide terminée par un contrepoids. Les deux bras de ce balancier sont de même longueur. La partie tube du balancier est reliée à un tube fixe de même diamètre par l'intermédiaire d'un manchon souple en caoutchouc. Ce tube fixe met en communication le déversoir oscillant avec le canal de décantation. 


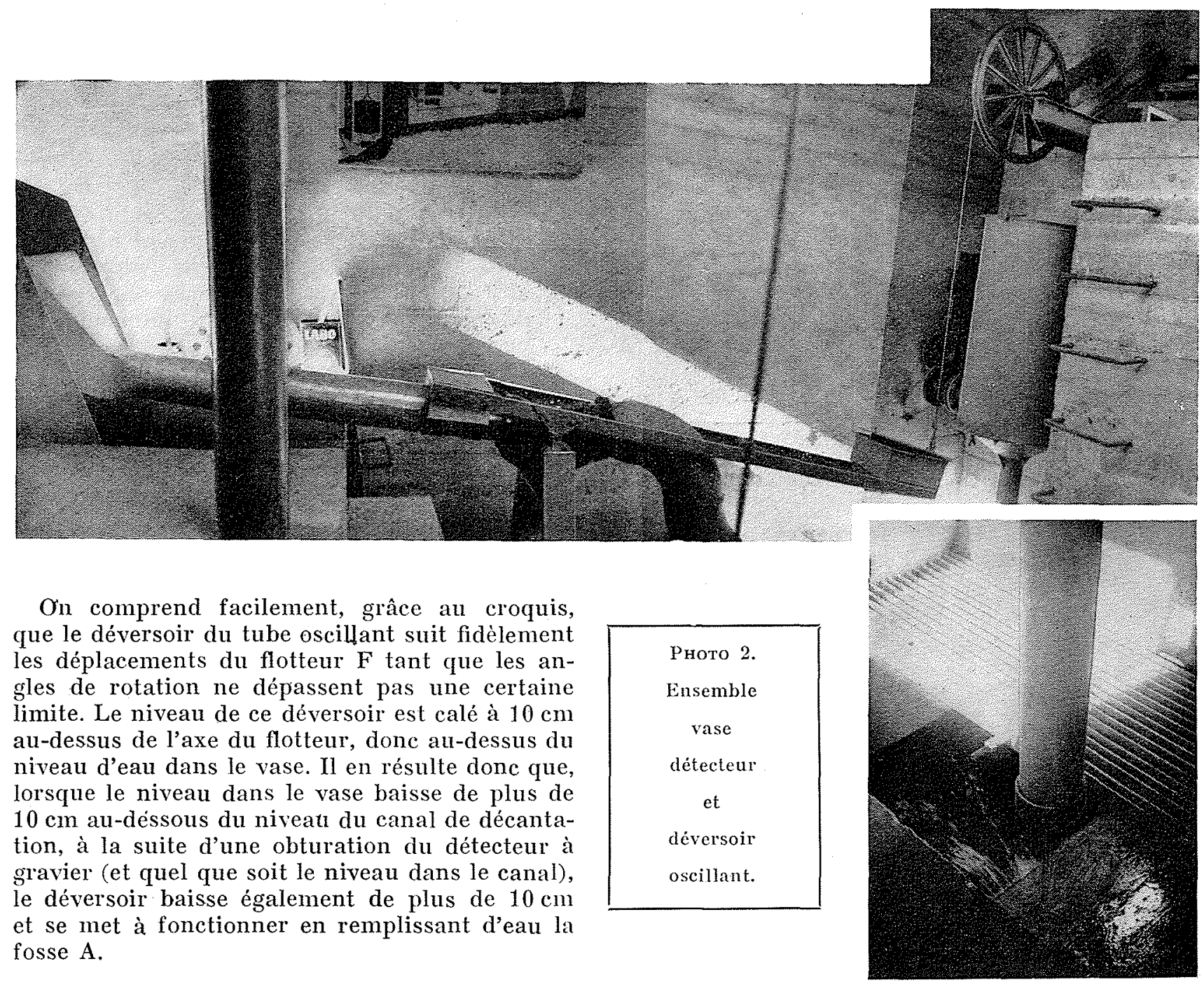

\subsubsection{3. - Siphon et vitesse d'ouverture de la vanne de chasse :}

Il est nécessaire d'utiliser un siphon qui se déclenche mêne avec un très faible débit d'amorcage. Nous avons trouvé le problème complètement résolu en utilisant les appareils automatiques de chasse d'eau pour égouts qui s'amorcent, grâce à un système pneumatique, non pas avec un débit d'amorcage, mais à partir d'une certaine charge d'eau. Ces appareils sont très robustes et ne nécessitent aucun entretien. La vitesse d'ouverture de la vanne secteur de chasse dépend du débit de ce siphon; si ce débit est trop fort, la vanne se lève très rapidement, provoquant dans le torrent une crue intempestive heureusement très courte. Il est bon, en conséquence, de calculer le débit du siphon pour que la vitesse de levée de la vanne de chasse soit telle que le débit de chasse - qu'elle laisse échapper pendant le temps où elle fonctionne encore en charge, n'excède pas le débit d'apport du torrent dans une proportion exagérée.

3.2.2.4. - Orifice de vidange du puits du flotteur. - Réglage de la durée des chasses (fig. 7) :

La vannette de chasse $C$ du puits du flotteur commande le temps de vidange de ce puits, donc la durée des chasses. Nous avons indiqué plus haut la manière dont cette durée peut être calculée. Notons, en outre, deux points de détail qui ont leur importance :

- Cette vannette ne doit pas être calée trop près du flotteur; il est bon de la caler au contraire à une distance $h=0,50 \mathrm{~m}$ à $1 \mathrm{~m}$ sous le fond du flotteur; ceci pour que le flotteur ait en-

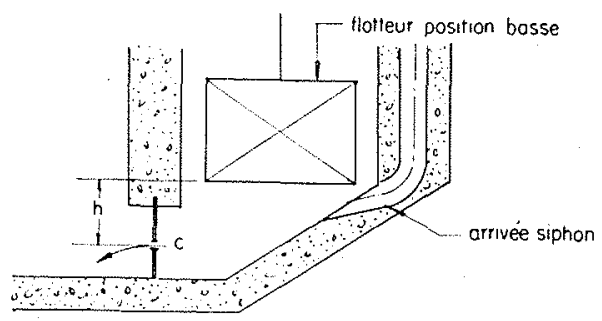

FIG. 7 


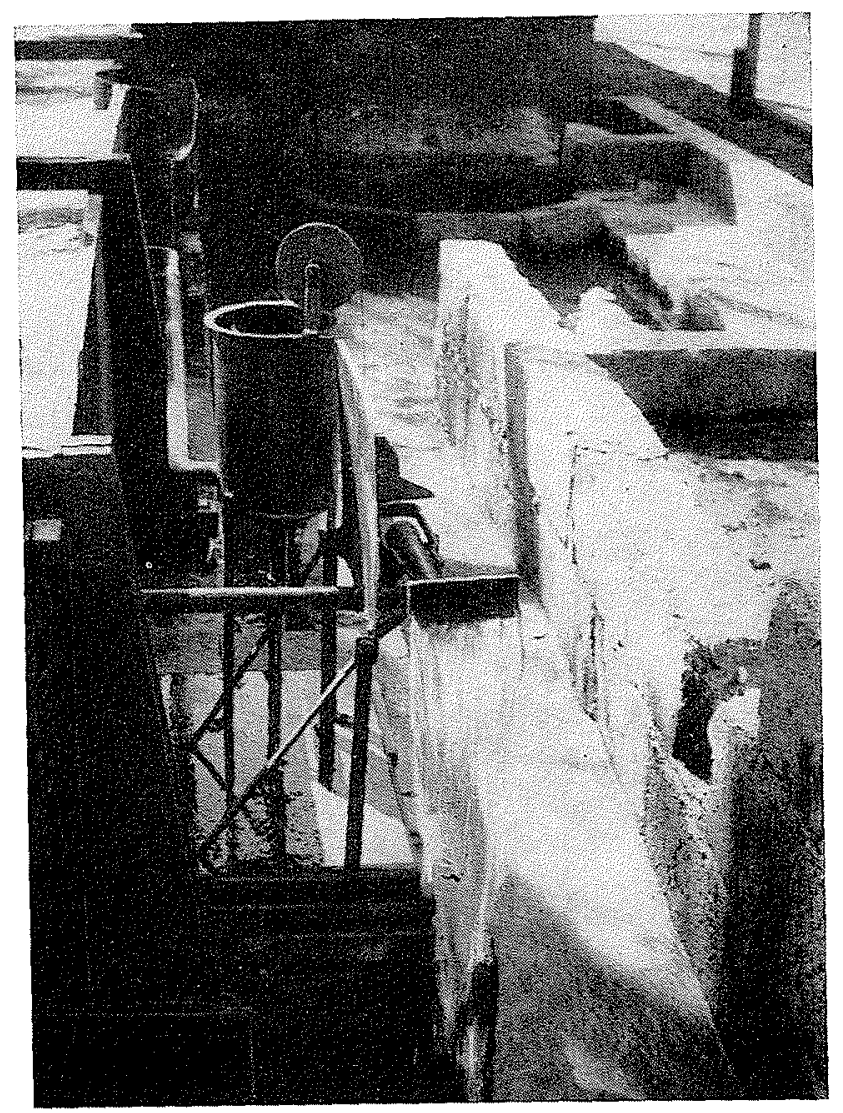

Pното $3 a^{\prime} a$ - Modèle réduit du dispositif de détection. core une vitesse appréciable au moment de la fermeture de la vanne. En effet, si celleci se ferme trop lentement, son étanchëité risque d'être moins bonne.

- L'expérience du Pingon nous a montré également qu'il était bon de remplacer cette vannette par un orifice calibré circulaire servant à régler la durée des vidanges de la vanne de chasse, la vannette servant uniquement au nettoyage de la fosse du flotteur.

\subsection{3. -- VANNES DE chasse :}

En principe, n'importe quelles vannes à secteur conviennent. Ces vannes peuvent être soit à surface libre, soit en charge (fig. 8).
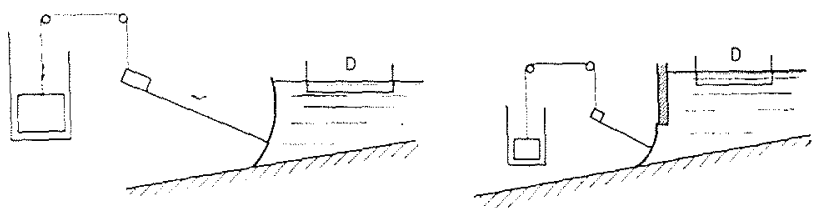

FIG. 8

La vanne en charge pose des problèmes techniques plus complexes, mais elle a l'avantage d'être beaucoup moins volumineuse et de permettre plus de souplesse dans la conception générale des ouvrages.

A Alpes-II, nous utilisons actuellement des vannes en charge d'un type un peu particulier qui

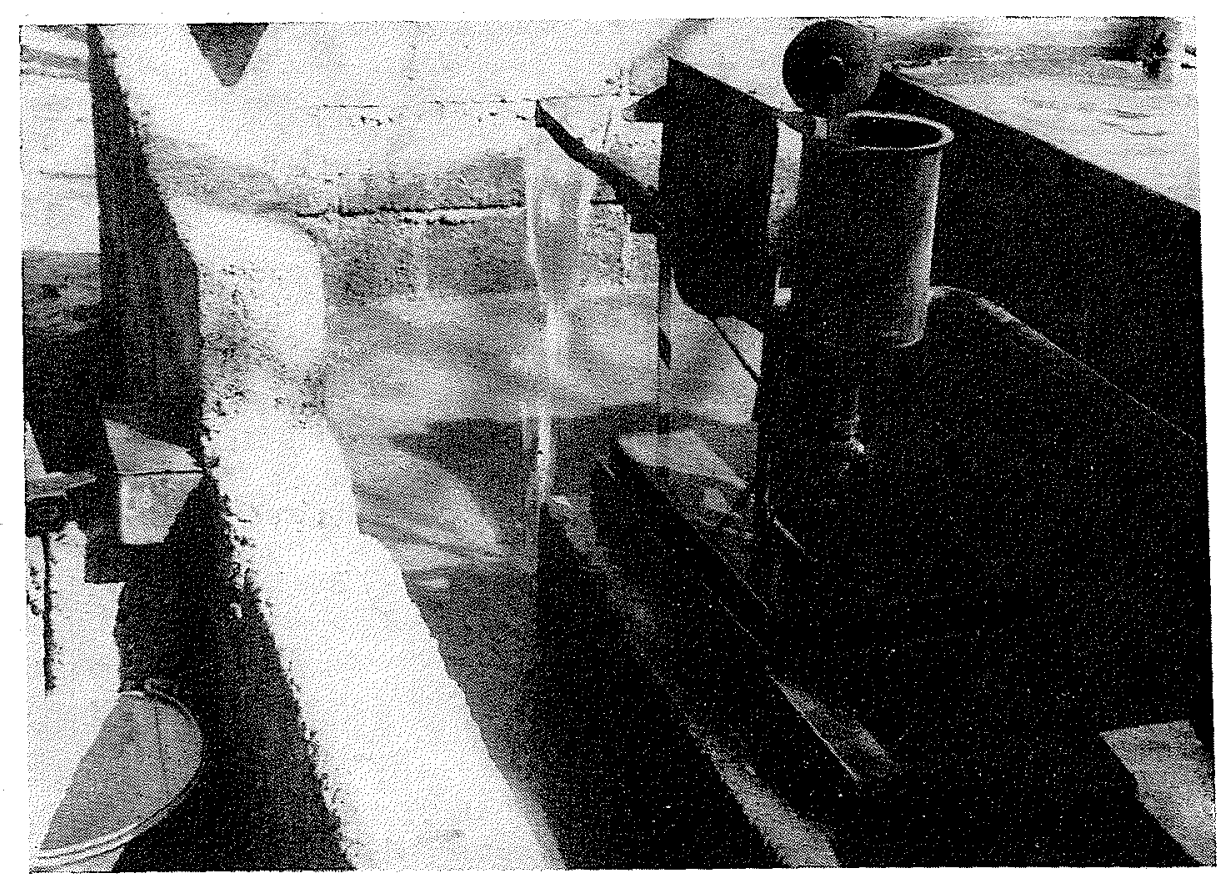

Pното $3 b$. - Modèle réduit du dispositif de détection, 
ont été étudiées et mises au point par M. Bourne, chef de Service au G.R.P.H. «Savoie » et dont le fonctionnement n'appelle aucune critique, tant par leur étanchéité que par la très faible valeur de leur frottement.

\section{3. - Références.}

\subsection{1. - Etudes Faites aU Laboratolre D'AlBertVille :}

L'ensemble dégraveur, détecteur à gravier, déversoir oscillant, a été mis au point sur modèle réduit au Laboratoire E.D.F. d'Albertville.

\subsection{2. - Prises D'EAU DÉJA RÉalisées aVEc UN DÉTECTEUR A GRAVIER.}

\subsubsection{1. - Prises d'eau du Grand-Pyx:}

La prise d'eau du Grand-Pyx fait partie de la chute d'Aussois. Cette prise dérive les eaux d'un torrent d'origine glaciaire qui a un charriage absolument énorme en été. La prise d'eau, réalisée en 1950 , quoique de conception très étudiée pour l'époque, avait causé de gros déboires à l'exploitation. Plusieurs fois cette prise s'était trouvée totalement ensablée à la suite de crues, ce qui avait eu pour conséquences des arrêts prolongés d'exploitation et par conséquent des pertes d'eau importantes, à tel point que le G.R.P.H. «Maurienne», qui exploite cette chute, a estimé rentable de refaire presque complètement cette prise d'eau en utilisant en particulier un détecteur à gravier.

Cette prise d'eau a fonctionné en automatique durant l'été 1957 et a donné entière satisfaction. Le G.R.P.H. envisage même de refaire certaines autres de ses prises d'eau sur ce type.

\section{- Prise de la Raja:}

L'aménagement de Roselend comportera 32 prises d'eau. Pour la plupart d'entre elles, la Région Alpes-II envisage d'utiliser le détecteur à gravier. Dans le but de vérifier le bon fonctionnement du détecteur et aussi et surtont de permettre certaines mises au point de détail, il a été décidé de construire tout de suite et de meltre en exploitation une petite prise, la «Raja», qui peut être dès maintenant dérivée dans un tronçon de galerie déjà en exploitation. Ceite prise d'eau a été exécutée en 1957; à la date où nous publions, elle a déjà subi avec succès les crues de fonte des neiges de mai et juin. On avait donné comme consigne, au personnel chargé de l'exploitation, de se contenter d'observer les appareils, sans jamais intervenir.

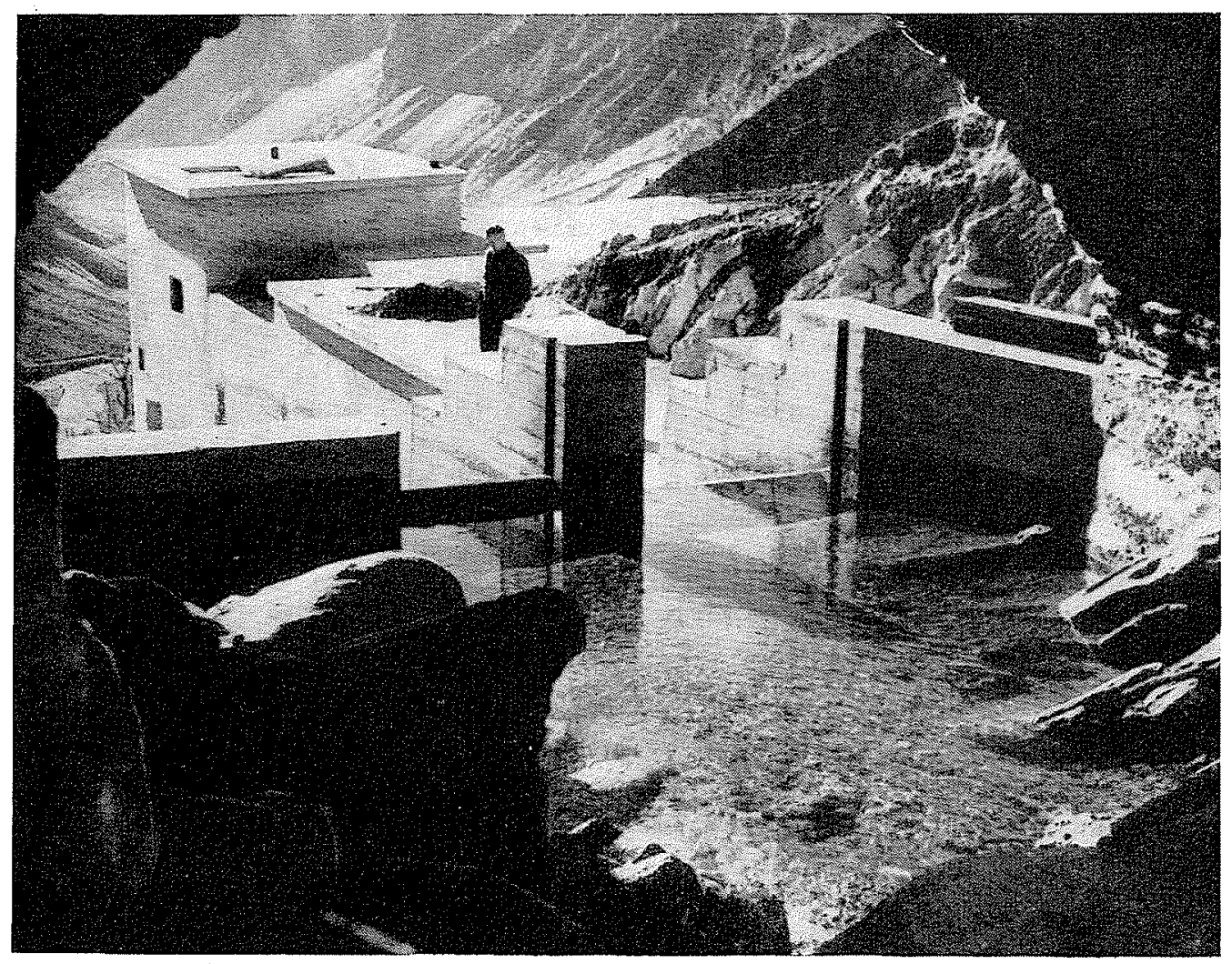

Рното 4. -- Prise d'eau de la Raja, 
Aucune panne n'a été constatée jusqu'à ce jour, et ce n'est pas faute d'avoir fonctionné, puisque, à certaines périodes, le détecteur a provoqué jusqu’à 3 ou 4 chasses par jour.

Etant donné que ce torrent est un des plus indisciplinés de tous ceux qu'on doit capter à Roselend, tant par la rapidité de ses crues que par la quantité de matériaux solides qu'il transporte, on peut, dès à présent, affirmer que le système marche bien, et que le risque de panne est extrêmement réduit.

\section{4. - CONSÉQUENCE DE L'ADOPTION DU DÉTECTEUR A GRAVIER. - MUR PLONGEANT}

Une prise d'eau automatique doit être pensée entièrement en fonction de cet automatisme, c'est-à-dire en tenant compte du fait que, pour de telles prises d'eau, le gardien viendra à périodes fixes assez éloignées, alors que, pour une prise d'eau non automatique, il est là cn permanence ou monte dès qu'il se passe quelque chose. Passons en revue les différentes parties d'un ensemble prise d'eau-dégraveur, dont la conception doit tenir compte de ce fait, en insistant particulièrement. sur un organe de protection mis au point à la Région et qui a été appelé «mur plongeant».

\section{1. - Prise alimentant directement une cen- trale, ou un canal risquant l'ensable- ment : utilité du mur plongeant.}

Toute prise d'eau, aussi bien conçue soit-elle, est susceptible d'avoir ce qu'on pourrait appeler des pannes. En particulier, au cours des crues exceptionnelles, il arrive même à une prise d'eau gardiennée de s'ensabler par suite du non-fonctionnement d'une vanne de chasse, ou pour toute autre raison. Ce risque, aussi faible soit-il, existe toujours. Et il est bien plus grave pour une prise sans gardien, car alors la panne ne peut être détectée et réparée qu'au cours de la visite périodique du gardien, ou (dans le cas où les conséquences sont sensibles à l'aval) assez longtemps après, puisque ces prises d'eau sont en général automatiques parce que difficiles d'accès.

Dans le cas où les prises d'eau alimentent un lac de retenue par l'intermédiaire d'une galerie à pente unique (ce qui est fort heureusement souvent le cas dans la conception actuelle des grands aménagements, du type Roselend), les conséquences d'une panne ne peuvent se traduire que par une perte d'ean, ou l'envoi d'une certaine quantité de transports solides dans la retenue, ce qui n'est pas très grave. Par contre, si cette prise alimente directement une usine ou un canal siphonnant, un envoi intempestif de sables et de graviers dans les conduites d'amenée risque d'avoir de sérieux inconvénients. Dans ce cas il nous a semblé indispensable de prévoir un organe supplémentaire appelé « mur plongeant » qui a pour mission (si le détecteur à gravier ne joue pas son rôle, ou si la vanne de chasse ne s'ouvre pas) de protéger les conduites d'amenée contre une arrivée de sable inopinée, en bouchant purement et simplement le passage de l'eau dérivée.

\subsection{1. - Principe du mur plongeant :}

Le mur plongeant n'est pas autre chose qu'un gros détecteur à gravier traversé par la totalité du débit dérivé (fig. 9).
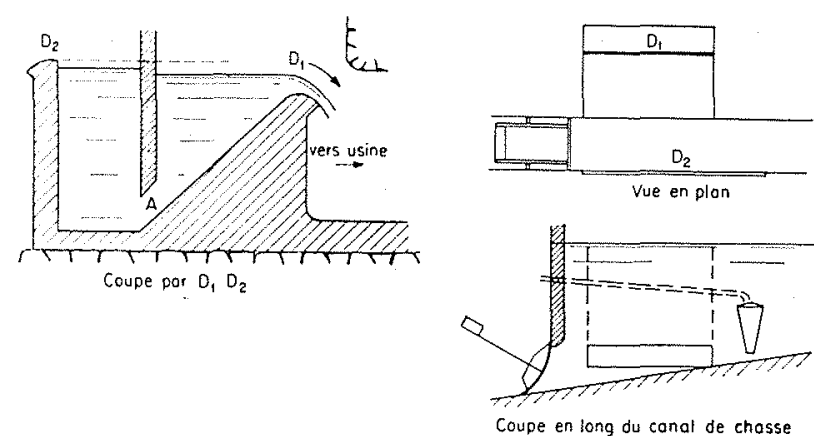

FrG. 9

Si la chasse, pour une raison quelconque, ne se produit pas, les graviers envahissent peu à peu la totalité du dessableur et commencent à boucher le mur plongeant. Il y a création d'une perte de charge en $\mathrm{A}$, le niveau monte dans le dessableur et une partie du débit s'écoule par D2 (déversoir de décharge). On conçoit que le phénomène s'amplifie rapidement, jusqu'à bouchage complet du mur plongeant et évacuation du débit entier par le déversoir D2.

Ce dispositif a été étudié systématiqement au laboratoire d'Albertville. Nous ne nous étendrons pas sur les étapes de ces essais; disons seulement qu'ils nous ont montré que, à condition de respecter certaines proportions, le fonctionnement en mur plongeant est absolument sûr, puisque entièrement statique. Le jeu des dimensions relatives - autrement dit: différence de cotes entre D1 et D2 et profondeur du mur - permet de calculer la taille maxima ou minima des cailloux qui boucheront le mur plongeant. Il est en effet inutile de faire fonctionner ce système avec des sables trop fins, étant 
donné le caractère exceptionnel de son fonctionnement; d'autre part, plus le sable bouchant est fin, plus les dimensions deviennent grandes.

\section{2. - Grilles et prises d'hiver.}

\subsection{1. - GRILLES DE PRISE D'EAU :}

Le problème des grilles en rivière nous a longtemps préoccupés. En effet, les grilles en rivière, surtout placées au fond du lit, se bouchent facilement avec les cailloux et rendent de ce fait peu intéressant un dessableur automatique. Il fallait trouver des grilles ne se bouchant pratiquement pas. Trois solutions sont possibles :

- Etudier des grilles sur lesquelles les cailloux glissent sans se coincer;

- Mettre les grilles d'une surface $n$ fois trop grande, de façon que, même bouchées partiellement, le débit passe.

- Ne pas mettre de grilles (voir Lapalisse) ou à défaut mettre des grilles très larges ne retenant que les cailloux d'une taille exceptionnelle.

C'est à cette dernière solution que nous nous sommes arrêtés; la pente des dégraveurs étant, bien entendu, largement suffisante pour évacuer en temps de crue tous les cailloux qui peuvent se présenter (1).

Quand nous projetons une prise d'eau située dans un torrent de réputation calme, on supprime purement et simplement les grilles en rivière; quand le torrent est du type Grand-Pyx, on met des grilles avec un écartement entre barreaux de 10 à $20 \mathrm{~cm}$. L'expérience prouve que la proportion de cailloux supérieure à 10 ou $20 \mathrm{~cm}$ de diamètre est infime et que de telles grilles restent très longtemps propres, et en tout cas suffisamment longtemps pour qu'elles ne soient pas sensiblement obturées entre deux visites périodiques du gardien.

(1) Pratiquement le détecteur a toutes les chances de faire la chasse avec la crue qui a amené les cailloux et c'est là un de ses avantages.

\subsection{2. - PRISES D'HIVER :}

En hiver, l'eau des torrents alpins est toujours propre au-dessus d'une certaine altitude. Les appareils d'automatisme sont alors superflus; il est même prudent de les déconnecter afin qu'ils ne risquent pas d'être détériorés par le gel.

Un problème se pose alors. A quelle période doit-on faire cette opération? En effet, en automne, il fait en général froid, mais la neige n'est pas encore là pour protéger et on est toujours à la merci d'un redoux intempestif qui amènerait la pluie et une crue, et qui pourrait causer des dégâts si les appareils d'automatisme étaient déconnectés. Disons tout de suite que la probabilité d'une telle aventure est très faible à partir du mois de novembre dans nos climats alpins. Néanmoins, elle existe. Nous avons donc pensé que la meilleure solution consistait à prévoir une prise d'hiver, calibrée de telle manière qu'aux faibles débits, le niveau d'eau dans le dessableur s'abaisserait de manière à mettre hors circuit les appareils d'automatisme. Aux forts débits, par contre (crue intempestive), les appareils d'automatisme seraient en service. Cette prise d'hiver est simplement constituée par un orifice situé à l'aval du détecteur (donc en principe à l'abri des cailloux) et court-circuitant le déversoir $D$ de dérivation (fig. 10).
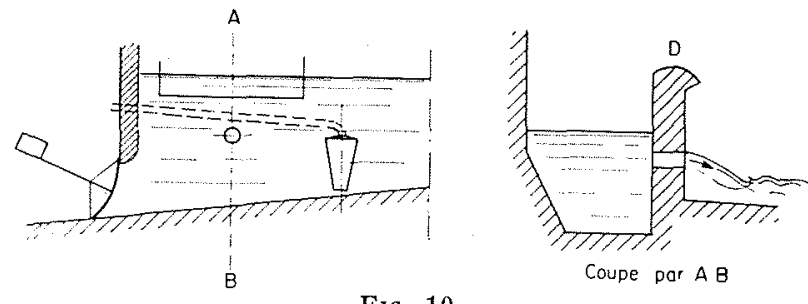

FIG. 10

Elle est, bien entendu, bouchée en été, car elle présente, malgré tout, un petit risque de laisser transiter les cailloux en galerie, risque qui peut être pris sans inconvénient l'hiver en raison de la très faible probabilité de fonctionnement en automatique.

\section{5. - PROPOSITION POUR UNE PRISE D'EAU TYPE}

Nous pensons que la meilleure manière de terminer cet exposé consiste à donner en exemple le plan d'une des futures prises d'eau de Rose- lend : la prise du Reclus, qui présente toutes les caractéristiques que nous avons exposées dans cet article et qui, à notre avis, peut être prati- 


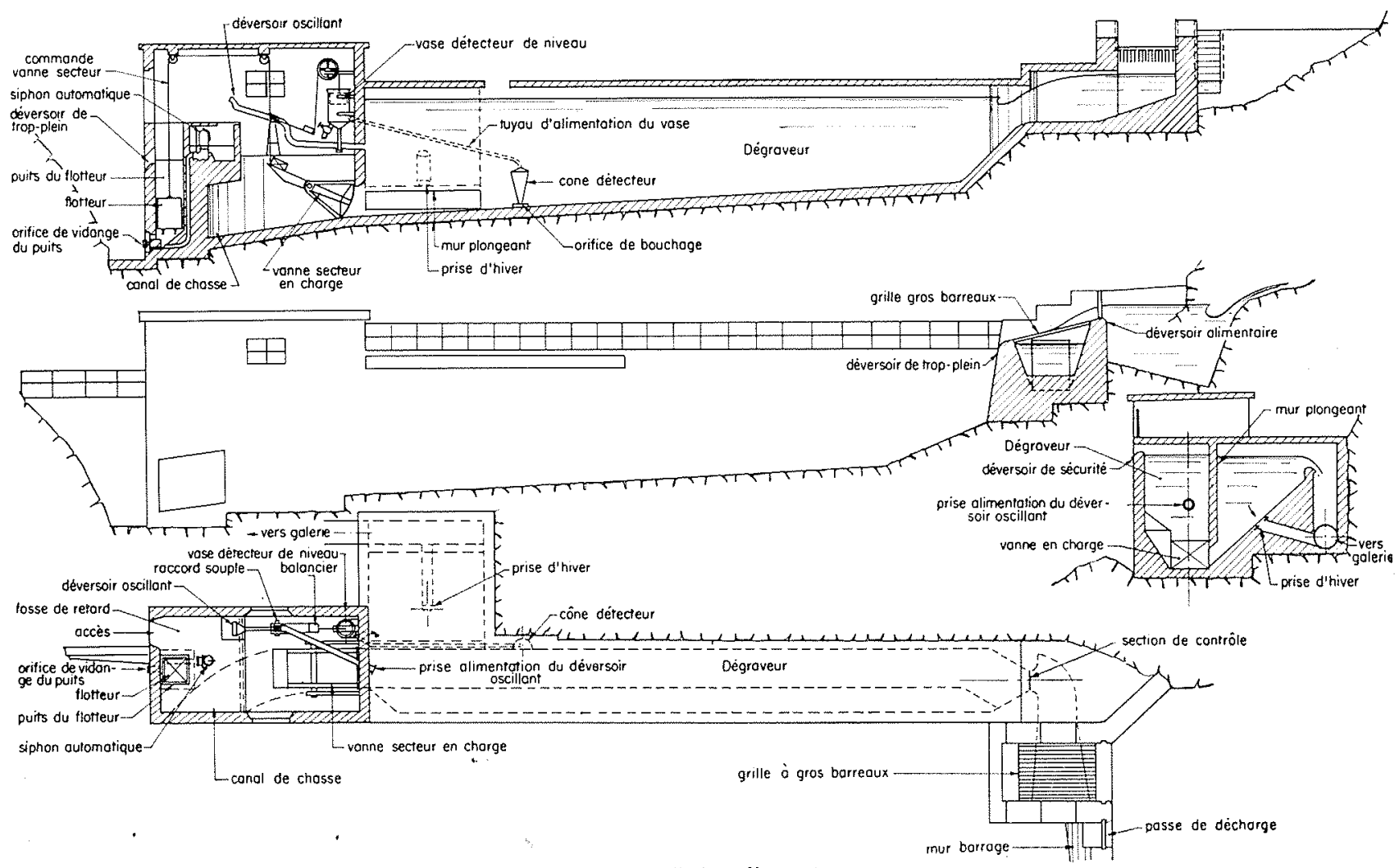

Fic. 11. - Prise d'eau type.

quement utilisée sur presque n'importe quel torrent (fig. 11).

Caractéristiques :

- B.V. $15 \mathrm{~km}^{2}$.

- Altitude des prises : 1800.
- Pas de glacier.

- Débit dérivé : $3 \mathrm{~m}^{3} / \mathrm{s}$.

- Alimente le lac de Roselend par l'intermédiaire d'une conduite se déversant dans la galerie d'amenée principale des adductions amont.

\section{Dis La Direction des Constructions et Armes Navales de Brest recherche :}

\section{INGÉNIEUR \\ CONTRACTUEL}

ayant bonnes connaissances: physique, acoustique ou hydraulique, électronique pour travaux recherche appliquée.

Adresser c.v, manuscrit et prétentions à :

D.C.A.N. BREST, Section Personnel 